\title{
ANALES DEL INSTITUTO DE LA PATAGONIA: LAS CIENCIAS NATURALES¹.
}

\author{
ANALES DEL INSTITUTO DE LA PATAGONIA: THE NATURAL SCIENCES
}

Vicente Pérez ${ }^{2}$

Los Anales del Instituto de la Patagonia tienen por objetivo principal la publicación de trabajos originales e inéditos realizados preferentemente por los investigadores de la Universidad de Magallanes, en los campos de las Ciencias Naturales referidos a la Patagonia, la Tierra del Fuego, la Antártica e islas adyacentes y el océano Pacífico sur-oriental

Ordenados alfabética y cronológicamente, se registran aquí 410 artículos y notas científicas publicados en los 35 volúmenes de los Anales del Instituto de la Patagonia, desde 1970 hasta 2007 inclusive, distribuidos en cinco áreas: Botánica (92), Ecología (55), Expediciones (6), Geociencias (69) y Zoología (191). El área Fundación (1) es introductoria y contiene la información sobre la fundación del Instituto de la Patagonia.

\section{FUNDACIÓN}

[Martinic, M.] 1979. Primer decenio del Instituto de la Patagonia. Cronología de una década. 10:251-263.

\section{BOTÁNICA}

Beeskow, A.M., M.A. Monsalve \& V. Duro 2005. Identificación de áreas de mayor diversidad en endemismos vasculares en la región patagónica argentina. 33:5-20.

Bjerke, J.W. \& A. Elvebakk 1999. Flavoparmelia soredians, a common but previously overlooked corticolous lichen species in southernmost Chile. 27:17-23.

Broughton, D.A., J.A. McAdam \& R. Branström 2000. A combined checklist and ecogeo- graphic conspectus for the vascular flora of Saunders Island, Falkland (Malvinas) Islands. 28:55-88.

Cornejo, S. 1996. Reporte preliminar de macroalgas presentes en bahía Laredo, estrecho de Magallanes. 24:87-95.

Dollenz, O. 1974. El Herbario del Instituto de la Patagonia. 5(1-2):122-125.

Dollenz, O. 1976. Números cromosómicos de Verbena tridens Lag., Baccharis patagonica Hook. et Arn. y Adesmia boronioides Hook. 7:163-167.

Dollenz, O. 1978. Números cromosómicos de espermatófitos de los géneros Berberis, Ribes y Festuca. 9:141-144.

Dollenz, O. 1979. Fitosociología de la vegetación fanerogámica halófita de bahía Dirección, estrecho de Magallanes. 10:169-177.

1 Perteneciente al proyecto Antecedentes para la Historia de las Ciencias Naturales en la Región de Magallanes

2 Laboratorio de Entomología, Instituto de la Patagonia, Universidad de Magallanes. Punta Arenas. vicente.perez@umag.cl 
Dollenz, O. 1980. Estudios fitosociológicos en el archipiélago del cabo de Hornos.11:225238.

Dollenz, O. 1981. Estudios fitosociológicos en el archipiélago cabo de Hornos. II. Relevamiento en la isla Hornos. 12:173-182.

Dollenz, O. 1981. Catálogo de las plantas vasculares de la Reserva Forestal Magallanes. .Lista preliminar correspondiente al área del cerro Mirador y río de las Minas. 12:205-21.

Dollenz, O. 1982. Estudios fitosociológicos en el archipiélago de cabo de Hornos. III. Relevamientos en la isla Deceit. 13:145-151.

Dollenz, O. 1982. Estudios fitosociológicos en las reservas forestales Alacalufes e isla Riesco. 13:161-170.

Dollenz, O. 1982. Fitosociología de la Reserva Nacional Magallanes. I. Estudio del área del cerro Mirador-río de las Minas. 13:171-181.

Dollenz, O. 1983. Fitosociología de la reserva forestal "El Parrillar", península de Brunswick, Magallanes. 14:109-118.

Dollenz, O. 1986. Relevamientos fitosociológicos en la península Muñoz Gamero, Magallanes. 18:51-53.

Dollenz, O. 1993 (1991). Sucesión vegetal en el sistema morrénico del glaciar Dickson, Magallanes, Chile. 20(1):49-60.

Dollenz, O. 1993 (1991). Capacidad de colonización de Rumex acetosella L. (vinagrillo) en comunidades perturbadas. 20(1):61-67.

Dollenz, O. \& A. Santana 2000. Clasificación y distribución espacial de la vegetación de cabo Negro (Magallanes, Chile) utilizando imágenes satelitales. 28:51-56.

Dollenz, O. 2003. Maytenus boaria Molina (Celastraceae) en la región de Magallanes, Chile. 31:87-89.

Domínguez, E. \& A. Elvebakk 2001. Lepidium draba L. (Brassicaceae), una adición a la flora adventicia de la región de Magallanes, Chile. 29:69-73.

Domínguez, E., A. Elvebakk, C. Muñoz, M. Uribe, C. Ríos \& B. López 2002. Gavilea kingii (Hook. F.) M.N. Correa. (Orchidaceae) en la región de Magallanes: confirmación de un registro histórico. 30:109-112.

Elvebakk, A. \& J.M. Henríquez 1994 (1993-1994). Contributions to the flora and vegetation of the Southern part of the Torres del Paine National Park, Última Esperanza, Chile. 22:33-42.

Gunckel, H. 1971 Las primeras plantas herborizadas en Chile en 1690. 2(1-2):134-141.

Guzmán, L. \& I. Campodonico 1972. Fitoplancton de red en puerto Edén, Chile ( 26 de marzo al 2 de abril de 1971). 3(1-2):209-219.

Henríquez, J.M., E. Pisano \& C. Marticorena 1995. Catálogo de la flora vascular de Magallanes (XII믈 Región, Chile). 23:6-30.

Henríquez, J.M. \& N. Caamaño 2002. Análisis de la flora vascular de valles glaciares de la región de Magallanes, Chile. 30:25-40.

Henríquez, J.M. \& N. Caamaño 2002. Estudios liquenométricos en el glaciar Nueva Zelandia, Tierra del Fuego: datos preliminares. 30:41-48.

Heusser, C.J., L.A. Borrero \& J.A. Lanata 1994 (1992). Late glacial vegetation at Cueva del Mylodon. 21:97-102.

Lembeye, G., L. Guzmán \& I. Campodonico 1978. Fitoplancton del sector oriental del estrecho de Magallanes, Chile (5 al 11 de abril de 1976). 9:221-228.

Looser, G. 1971. Botrychium lunaria (Pteridophyta) en Patagonia y Tierra del Fuego. Especie de área bipolar. 2(1-2):142-144.

Mansilla, A., C. Werlinger \& N. Navarro 2002. Efecto de fotoperíodo $e$ intensidad luminosa sobre el desarrollo de tetrásporas y carpósporas de Mazzaella laminarioides (Bory) Frederiq (Gigartinales-Rhodophyta). 30:49-60.

Mansilla, A., M. Palacios \& S. Aguilar 2004. Efecto de la salinidad en el desarrollo inicial de Sarcothalia crispata (Bory) Leister (Rhodophyta, Gigartinales) bajo condiciones de laboratorio. 32:13-23.

Martinic, M. 1982. Antecedentes para el conocimiento botánico y zoológico de la región del estrecho de Magallanes. 13:113-123.

Matteri, C. 1973. Notas briológicas (Musci) I. 4(13):273.

Matteri, C. 1973. Notas briológicas (Musci) II. 4(13): 274 .

Matteri, C. \& M.R. Piñero 1987. Sinopsis de las Rhizogoniaceae (Musci) en las regiones fueguina y patagónica. 17:59-65. 
Moore, D.M. 1974. Catálogo de las plantas vasculares nativas de Tierra del Fuego. 5(12):105-121.

Moore, D \& R.N. Goodall 1977. La flora adventicia de Tierra del Fuego. 8:263-274.

Palacios, M. \& A. Mansilla 2003. Desarrollo de gametófitos y esporófitos de Macrocystis pyrifera (L.) C. Agardh (Laminariales: Lessoniaceae) de la región de Magallanes en condiciones de laboratorio. 31:43-53.

Pisano, E. 1970. Vegetación del área de los fiordos Toro y Cóndor y puerto Cutter Cove. 1(1):27-60.

Pisano, E. 1971. Comunidades vegetales del área del fiordo Parry, Tierra del Fuego (Parque Nacional "Alberto M. de Agostini"). 2(12):93-133.

Pisano, E. 1972. Comunidades vegetales del área de bahía Morris, isla Capitán Aracena, Tierra del Fuego (Parque Nacional "Hernando de Magallanes"). 3(1-2):97-130.

Pisano, E. 1972. Algunos resultados botánicos de la expedición neozelandesa al Hielo Nor-Patagónico 1971-72. 3(1-2):131-160.

Pisano, E. 1973. Fitogeografía de la península de Brunswick, Magallanes. 4(1-3):141306.

Pisano, E. 1975. Ampliación del área distribucional de Dacrydium fonckii (Phil.), Florin. 6(12):139-140.

Pisano, E. 1976. Cormófitos acuáticos de Magallanes. 7:116-136.

Pisano, E. 1976. Extensión del área distribucional de Blechnum chilense (Kaulf) Mett. (Pteridophyta, Blechnaceae). 7:137-138.

Pisano, E. 1976. Adiciones a la flora vascular nativa de Tierra del Fuego. 7:155-157.

Pisano, E. 1976. Nuevos taxa para la flora vascular Fuego-Patagónica austral. 7:159-162.

Pisano, E. 1977. Contribución de Enrique Ibar Sierra al conocimiento de la naturaleza de la Patagonia oriental austral. 8:95-119.

Pisano, E. 1977. Fitogeografía de Fuego-Patagonia chilena I.- Comunidades vegetales entre las latitudes $52^{\circ}$ y $56^{\circ} \mathrm{S}$. 8:121-250.

Pisano, E. 1978. Establecimiento de Nothofagus betuloides (Mirb.) Blume (coigüe de Magallanes) en un valle en proceso de desglaciación. 9:107-128.
Pisano, E. 1978. Extensión del área distribucional de Asplenium obliquum Forst. var. sphenoides (Kunze) Espinoza (Pteridophyta, Aspleniaceae). 9:129-131.

Pisano, E. 1979. Fuchsia magellanica Lam. var. eburnea var. nov. 10:155-162.

Pisano, E. 1979. Presencia de Plantago tehuelcha Speg. en Chile. 10:163-166.

Pisano, E. 1979. Dacrydium fonckii (Phil.) Florin (Podocarpaceae), nueva especie para la flora fueguina. 10:167-168.

Pisano, E. 1980. Catálogo de la flora vascular del archipiélago del cabo de Hornos. 11:151-189.

Pisano, E. 1980. Distribución y características de la vegetación del archipiélago del cabo de Hornos. 11:191-224.

Pisano, E. 1981. Bosquejo fitogeográfico de FuegoPatagonia. 12:159-171.

Pisano, E. \& R.P. Schlatter 1981. Vegetación y flora de las islas Diego Ramírez (Chile). I. Características y relaciones de la flora vascular. 12:183-194.

Pisano, E. \& R.P. Schlatter 1981. Vegetación y flora de las islas Diego Ramírez (Chile). II. Comunidades vegetales vasculares. 12:195-204.

Pisano, E. 1982. Comunidades vegetales de la isla Hornos (archipiélago del Cabo de Hornos, Chile). 13:125-143.

Pisano, E. 1982. Adiciones a la flora vascular del archipiélago del cabo de Hornos. 13:153159.

Pisano, E. 1983. Comunidades vegetales en el sector norte de la península Muñoz Gamero (Última Esperanza, Magallanes). 14:83-101.

Pisano, E. \& R. Rossow 1983. Saxifraga umbrosa L., nueva especie adventicia para Chile. 14:103-107.

Pisano, E. 1984. Relaciones circumantárticas de la flora vascular del archipiélago del cabo de Hornos. 15:5-24

Pisano, E. \& C. Venegas 1984. Primera colección botánica y esquema fitogeográfico de la isla Noir, Tierra del Fuego. 15:25-35.

Pisano, E. 1986 (1985-1986). Flora vascular del sector terminal del fiordo Peel, Última Esperanza, Chile. 16:5-20.

Pisano, E. 1986 (1985-1986). Especies fuego-patagónicas de Grammitis Swartz. (Grammitidaceae, Pteridophyta). 16:21-49. 
Pisano, E. 1986 (1985-1986). Nueva colección referida a Taraxacum rhusiocarpum Dahlst. 16:51-53

Pisano, E. 1986 (1985-1986). Sectorización fitogeográfica del archipiélago sud patagónicofueguino: I. Vegetación del valle periglacial del río Murtillares, fiordo Peel, Última Esperanza, Chile. 17:5-57.

Pisano, E. 1987. Extensión del área distribucional de Poa darwiniana Parodi (Gramineae-Festuceae). 17:67-68.

Pisano, E. 1990 (1988). Sectorización fitogeográfica del archipiélago sud patagónico-fueguino. II: Vegetación y flora vascular del área del Parque Nacional Laguna San Rafael, Aisén (Chile). 18:5-34.

Pisano, E. 1991 (1989-1990). Sectorización fitogeográfica del archipiélago sud patagónico-fueguino. III: relaciones florísticas. 19(1):5-16.

Pisano, E. 1993 (1991). Sectorización fitogeográfica del archipiélago sud patagónico-fueguino. IV: afinidades florísticas y fitogeográficas entre 30 localidades. 20(1):7-34.

Pisano, E. 1993 (1991). Polygala darwiniana A.W. Bennett, nueva especie para la flora de Chile. 20(1):35-38.

Pisano, E. 1993 (1991). Nueva colección de Catabrosa aquatica (L.) P. Beauv. (Gramineae, Festuceae) en Tierra del Fuego e interpretación de su expansión de Rango. 20(1):39-47.

Pisano, E. 1994 (1992). Sectorización fitogeográfica del archipiélago sud patatagónico- fueguino. V: Sintaxonomía y distribución de las unidades de vegetación vascular. 21:5-33.

Pisano, E. \& J.M. Henríquez 1994 (1992). Xerodraba patagonica (Speg.) Skottsb. (Cruciferae) nueva especie para la flora de Chile. 21:35-38.

Pisano, E. \& J.M. Henríquez 1994 (1993-1994). Antecedentes botánicos sobre el cordón Baquedano, Tierra del Fuego, Chile. 22:5-19.

Pisano, E. 1996. Corsiaceae, nueva familia para la flora vascular de Magallanes y una nueva especie para la flora de Chile. 24:5-13.

Pisano, E. 1996. Implicaciones de la teoría de biogeografía de islas en el diseño de reservas naturales. 24:117-126.

Pisano, E. 1998 (1997). Los bosques de Patagonia austral y Tierra del Fuego chilenas. 25:919.
Podestá, F., V. Fajardo \& A. Urzúa 1987. Constituyentes químicos de Berberis buxifolia Lam. 17:99-102.

Rojas, G. 1998. Vegetación, potencialidad alimentaria y utilitaria para el indígena de Tres Arroyos, Tierra del Fuego, Chile. 26:91-99.

Rozzi, R., F. Massardo, J. Silander, O. Dollenz, B. Connolly, C. Anderson \& N. Turner 2003. Árboles nativos y exóticos en las plazas de Magallanes 31:27-42.

Teneb, E. \& O. Dollenz 2004. Distribución espacial de la flora vascular, la humedad y el pH en un turbal de esfagno (Sphagnum magellanicum Brid.), Magallanes, Chile. 32:5-12.

Tuhkanen, S. I. Kuokka, J. Hyvönen, S. Stenroos \& J. Niemelä 1992 (1989-1990. Tierra del Fuego as a target for biogeographical research in the past and present 19(2):5-107.

Wheeler, G.A. 1986 (1985-1986). Carex minutissima Barros (Cyperaceae) nueva para Chile y Argentina meridionales. 16:63-65.

Wheeler, G.A. 1994 (1993-1994). The Uncinia (Cyperaceae) of Tierra del Fuego, the Falkland Islands and South Georgia. 22:21-31.

Wheeler, G.A. 1995. The Uncinia (Cyperaceae) of Tierra del Fuego, the Falkland Islands and South Georgia. Appendix. 23:31-34.

\section{ECOLOGÍA}

Andrade, C. \& C. Ríos 2007. Estudio experimental de los hábitos tróficos de Trophon geversianus (Pallas 1774) (Gastropoda: Muricidae): selección y manipulación de presas 35(1):45-54.

Armesto, J., M.K. Arroyo \& A. Peñaloza 1996. Condiciones para la sustentabilidad ecológica del manejo de bosques y el proyecto "Río Cóndor". 24:29-39.

Atalah, A., W. Sielfeld \& C. Venegas 1980. Antecedentes sobre el nicho trófico de Canis griseus en Tierra del Fuego. 11:259-271.

Briones, M., R. Schlatter, W. Wolodarsky \& C. Venegas 2001. Clasificación ambiental para hábitat de Castor canadensis (Kuhl, 1820, Rodentia), de acuerdo a características de cuencas en un sector de Tierra del Fuego. 29:75-93.

Brown, C.E., C.B. Anderson, S. Ippi, M.F. Sherriffs, R. Charlín, S. McGehee \& R. Rozzi 2007. The 
autoecology of the fío-fío (Elaenia albiceps Lafresnaye \& D'Orbigny) in Subantarctic forests of the Cape Horn Biosphere Reserve, Chile. 35(2):29-40.

Burry, L.S., M.E. Trivi de Mandri \& H.L.D'Antoni 2007. Modern analogues and past environments in Central Tierra del Fuego, Argentina. 35(2):5-14.

Campodonico, I. \& L. Guzmán 1974. Marea roja producida por Amphidoma sp. en el estrecho de Magallanes. 5(1-2):209-213.

Campodonico, I., L. Guzmán \& G. Lembeye 1975. Una discoloración causada por el ciliado Mesodinium rubrum (Lohmann) en ensenada Wilson, Magallanes. 6(1-2):225-239.

Campodonico, I. 1977. Algunos aspectos biológicopesqueros del recurso centollón Paralomis granulosa (Jacquinot) (Decapoda, Anomura, Lithodidae) en el estrecho de Magallanes. 8:363-373.

Campodonico, I. \& L. Guzmán 1977. Una relación interespecífica entre un pez lipárido y el crustáceo Lithodes antarctica. 8:389-390.

De los Ríos, P. \& N. Rivera 2007. Branchinecta (Branchiopoda, Anostraca) as bioindicator of oligotrophic and low-conductivity shallow water bodies in southern Chilean Patagonia. 35(2)15-20.

Dollenz, O. 1977. Estado de la flora vascular en puerto Espora, Tierra del Fuego, contaminada por el petróleo del B/T "Metula". I.- Reconocimiento de la entrada de mar noroeste. 8:251-261.

Dollenz, O. 1978. Estado de la flora vascular en puerto Espora, Tierra del Fuego, contaminada por el petróleo del B/T "Metula". II.- Reconocimiento de la entrada de mar suroeste. 9:133-139.

Dollenz, O. \& J. Ivanovic 1996. Sucesión secundaria en un pastizal incendiado en el Parque Nacional Torres del Paine, Magallanes, Chile. 24:15-28.

Domínguez, E., E. Pisano \& O. Dollenz 1999. Colonización vegetal en el área periglacial del glaciar Nueva Zelandia, cordillera Darwin de Tierra del Fuego, Chile. 27:7-16.

Eberhard, P. 2006. Nueva regulación sobre responsabilidad ambiental en la Antártica. 34:91-93.
Fabiano, M. \& C. Misic 1995. Biochemical composition of organic detritus during degradation of Euphausia vallentini (Stebbing). 23:101-110.

Faúndez, E. 2005. Asociación críptica de Gayomyia falcata (Blanchard, 1851) (Neuroptera: Hemerobiidae) con la zarzaparrilla Ribes magellanicum Poiret, 1812 (Saxifragaceae) en la región de Magallanes. 33:63-64.

Faúndez, E.I. 2007. Notes on the biology of Ditomotarsus punctiventris Spinola, $1852(\mathrm{He}-$ miptera: Acanthosomatidae) in the Magellan region, and comments about the crypsis in Acanthosomatidae. 35(2):67-70.

Ferreyra, M.V., D. Grigera \& C. Úbeda 2005. Conservación de los ecosistemas de alta montaña: la zona altoandina del Parque Nacional Nahuel Huapi (Argentina). 33:41-58.

Fuentealba, R. J. Lozic \& A. Zegpi 1981. Observaciones clínicas de una intoxicación masiva con veneno paralítico de los mariscos (Puerto Natales, Magallanes, Chile). 12:289-293.

García-Tello, P., V. Campos \& C. Riquelme 1980. Observaciones en microflora epífita y su alteración por efecto de la contaminación con petróleo. 11:333-336-

Guzmán, L. I. Campodonico \& J. Hermosilla 1975. Estudio sobre un florecimiento tóxico causado por Gonyaulax catenella en Magallanes. I. Distribución espacial y temporal de G. catenella. 6(1-2):173-183.

Guzmán, L. \& G. Lembeye 1975. Estudio sobre un florecimiento tóxico causado por Gonyaulax catenella en Magallanes. II. Algunas condiciones hidrográficas asociadas. 6(12):185-195.

Guzmán, L., I. Campodonico \& M. Antunovic 1975. Estudio sobre un florecimiento tóxico causado por Gonyaulax catenella en Magallanes. IV. Distribución y niveles de toxicidad del veneno paralítico de los mariscos (noviembre de 1972, noviembre de 1973). 6(1-2):225-239.

Guzmán, L. 1976. Estudio sobre un florecimiento tóxico, causado por Gonyaulax catenella en Magallanes. V.- El probable ciste ecdísico de G. catenella. 7:201-206.

Guzmán, L. \& C. Ríos 1981. Estructura de macroorganismos de una playa de bloques y cantos de isla Wollaston, archipiélago del cabo de Hornos. 12:257-271. 
Jaksic, F., J. Rau \& J. Yáñez 1978. Oferta de presas y predación por Bubo virginianus (Strigidae) en el Parque Nacional "Torres del Paine". 9:199-202.

Koch, J. \& R. Kilian 2002. Dendroecological potential of common tree species along a transect across the southernmost Andes, Chile (53으. 30:123-132.

Lanfranco, D. 1979. Situación de la fauna entomológica en puerto Espora, Tierra del Fuego, luego de la contaminación provocada por el petróleo del B/T "Metula". I. Entomofauna suelo-superficial. 10:209-218.

Langley, S. \& G. Lembeye 1977. Algunos antecedentes sobre el macrobentos, granulometría y contenido de petróleo en los sedimentos de dos entradas de mar en puerto Espora (Tierra del Fuego) contaminados por el derrame del B/T "Metula". 8:375-388.

Lecaros, O. \& P. Mackenney 1981. Determinaciones preliminares de mercurio en crustáceos litódidos y peces marinos de la región magallánica. 12:295-299.

Lecaros, O., M. Quilodrán \& M.S. Astorga 1990 (1988). Determinación de hidrocarburos parafínicos derivados del petróleo del B/T "Cabo Pilar" en el estrecho de Magallanes. 18:113-125.

Lembeye, G., L. Guzmán \& I. Campodonico 1975. Estudio sobre un florecimiento tóxico causado por Gonyaulax catenella en Magallanes. III. Fitoplancton asociado. 6(1-2):197-208.

Lembeye, G. 1981. Segunda aparición del veneno paralítico de los mariscos (VPM) asociado a Gonyaulax catenella, en Magallanes (Chile). 12:273-276.

Lembeye, G. 1981. Estructura del fitoplancton asociado a la presencia del veneno paralítico de los mariscos en seno Unión y áreas adyacentes (Magallanes, Chile). 12:277-288.

McGehee, S.M. 2007. Consumption of leñadura ( $M a-$ ytenus magellanica) seeds by three primarily insectivorous bird species. 35(1):71-74.

Mc Nutt, J. 1981. Selección de presa y comportamiento de caza del halcón peregrino (Falco peregrinus) en Magallanes y Tierra del Fuego. 12:221-228.

Moore, D.M. \& E. Pisano 1998 (1997). Biotic colonization of recently deglaciated areas in
Fuego-Patagonia: phytogeographical considerations. 25:21-46.

Moorman, M.C., C.B. Anderson, A.G. Gutiérrez, R. Charlín \& R. Rozzi 2006. Watershed conservation and aquatic benthic macroinvertebrate diversity in the Alberto D'Agostino National Park, Tierra del Fuego, Chile. 34:41-58.

Pisano, E. 1971. Estudio ecológico preliminar del Parque Nacional "Los Pingüinos" (estrecho de Magallanes). 2(1-2):94-133.

Pisano, E. 1972. Observaciones fito-ecológicas en las islas Diego Ramírez. 3(1-2):161-169.

Pisano, E. \& M.J. Dimitri 1973. Estudio ecológico de la región continental sur del área AndinoPatagónica. 4(1-3):207-271.

Pisano, E. 1974. Estudio ecológico de la región continental sur del área Andino-Patagónica. II. Contribución a la fitogeografía de la zona del Parque Nacional "Torres del Paine". 5(12):59-104.

Pisano, E. 1975. Características de la biota derivada de factores especiales. 6(1-2):123-137.

Pisano, E. 1976. Contaminación por petróleo del $\mathrm{B} / \mathrm{T}$ "Metula" en vegetación fanerogámica litoral. 7:139-153.

Pisano, E. 1991 (1989-1990). Labilidad de los ecosistemas terrestres fuego-patagónicos. 19(1):17-25.

Ríos, C. \& L. Guzmán 1982. Reevaluación de la estructura de la comunidad en una playa de bloques y cantos de alta latitud (archipiélago del cabo de Hornos). 13:211-224.

Rozzi, R., R. Charlín, S. Ippi \& O. Dollenz 2004. Cabo de Hornos: un Parque Nacional libre de especies exóticas en el confín de América. 32:55-62.

Rozzi, R., F. Massardo, A. Mansilla. C. Anderson, A. Berghöfer, M. Mansilla, M.R. Gallardo, J. Plana, U. Berghöfer, X. Arango, S. Russell, P. Araya \& E. Barros 2007. La Reserva de Biosfera Cabo de Hornos: un desafío para la conservación de la biodiversidad e implementación del desarrollo sustentable en el extremo austral de América. 35(1):55-70.

Santana, M. \& J. Cañete 2001. Antecedentes biológicos para el cultivo del caracol marino Trophon geversianus (Pallas 1774) (Gastropoda: Muricidae) en Magallanes. 29:225-115. 
Sielfeld, W. \& C. Venegas 1980. Poblamiento e impacto ambiental de Castor canadensis Kuhl, en isla Navarino, Chile. 11:247-257.

Uribe, J.C. 1990 (1988). Antecedentes sobre un tercer brote de veneno paralizante de moluscos (VPM) en la región de Magallanes. 18:97-101.

Uribe, J.C. 1990 (1988). Observaciones sobre algunos fenómenos recurrentes en el fitoplancton de seno Unión y bahía Bell (región de Magallanes) y su relación con la estabilidad de la columna de agua. 18:103-111.

Venegas, C. \& R. Schlatter 1999. Efecto de la intervención silvícola en bosques de Nothofagus pumilio sobre ensambles avifaunísticos estivales en Tierra del Fuego (Chile). 27:41-50.

\section{EXPEDICIONES}

Gunckel, H. 1971. Las primeras plantas herborizadas en Chile en 1690. 2(1-2):134-141.

Martinic, M.1982. Antecedentes para la historia del conocimiento botánico y zoológico de la región del estrecho de Magallanes. 13:113-123.

Pisano, E. 1972. Algunos resultados botánicos de la expedición neozelandesa al Hielo Nor-Patagónico, 1971-72. 3(1-2):131-160.

Pisano, E. 1977. Contribución de Enrique Ibar Sierra al conocimiento de la naturaleza de la Patagonia oriental austral. 8:95-119.

Tuhkanen, S., I. Kuokka, J. Hyvönen, S. Stenroos \& J. Niemelä 1992 (1989-1990). Tierra del Fuego as a target for biogeographical research in the past and present. 19(2):5-107.

Tuhkanen, S. 1998 (1997). La Tierra del Fuego como objeto de investigación para investigadores finlandeses de la naturaleza. 25:105-116.

\section{GEOCIENCIAS}

Andrade, S.G. 1993 (1991). Geomorfología costera y antecedentes oceanográficos físicos de la región de Magallanes, Chile. 20(1):135-151.

Araos, J., M.A. Godoi \& R. Carvallo 2007. Adelgazamiento y dinámica en la zona de ablación del glaciar Tyndall, Campo de Hielo Patagónico Sur. 35(1):19-28.
Araya, J. 2000. Perfiles submarinos por los piedmonts del estrecho de Magallanes y bahía Nassau, Chile austral. 28:23-40.

Butorovic, N. 2001. Resumen meteorológico año 2000. Estación "Jorge C. Schythe". 29:173182.

Butorovic, N. 2002. Resumen meteorológico año 2001. Estación "Jorge C. Schythe" (530's; 7053’W; 6 m s.n.m.). 30:157:164.

Butorovic, N. 2003. Resumen meteorológico año 2002. Estación "Jorge C. Schythe" (5308'S; 7053'; 6 m s.n.m.). 31:123-130.

Butorovic, N. 2004. Resumen meteorológico año 2003. Estación "Jorge C. Schythe" (5308'S; 7053'O; 6 m s.n.m.). 32:79-86.

Butorovic, N. 2005. Resumen meteorológico año 2004. Estación "Jorge C. Schythe" (5308'S; 7053'W; 6 m s.n.m.). 33:65-71.

Cañón, A. 2000. Nuevos antecedentes en la estratigrafía de la Cuenca de Magallanes. 28:41-50.

Carrasco, J.F., G. Casassa \& A. Rivera 1998. Climatología actual del campo de Hielo Sur y posibles cambios por el incremento del efecto invernadero. 26:119-128.

Casassa, G. \& A. Rivera 1998. Digital radioecho sounding at Tyndall glacier, Patagonia. 26:129-135.

Casassa, G., A. Rivera, M. Aniya \& R. Naruse 2000. Características glaciológicas del Campo de Hielo Patagónico Sur. 28:522.

Casassa, G., V. Damm, D. Eisenburger, M. Jenett, C. Cárdenas, C. Acuña, A. Rivera \& H. Lange 2001. Estudios glaciológicos en Patagonia y Chile central utilizando un sistema aerotransportado de radio ecosondaje. 29:25-44.

Coronato, F. 1994 (1992). Influencia de las mesetas del Este de la Patagonia central en las características oceánicas del clima del área. 21:131-146.

Coronato, F. 1995. El viento como modificador de la percepción de las temperaturas en el sur de la Patagonia. 23:111-118.

Endichler, W. \& A. Santana 1990 (1988). El clima del sur de la Patagonia y sus aspectos ecológicos. Un siglo de mediciones climatológicas en Punta Arenas. 18:57-86. 
Endlicher, W. \& A. Santana 1998 (1997). El invierno de 1995: un fenómeno climático muy severo en la Patagonia austral. 25:77-88.

Godoi, M.A., G. Casassa \& T. Shiraiwa 2001. Reseña de estudios paleoclimáticos mediante testigos de hielo: potencialidades y evidencia obtenida en el cono sur de Sudamérica. 29:45-54.

Godoi, M.A., R. Carvallo \& M. Arévalo 2003. Condición actual del lóbulo Zapata Sur, en la zona de ablación del glaciar Tyndall, mediante radio-ecosondeo sobre la superficie glaciar. 31:5-15.

Heusser, C.J., L.E. Heusser \& A. Hauser 1991 (1989-1990). A 12000 yr B.P. tephra layer at Bahía Inútil (Tierra del Fuego, Chile). 19(1):339-49.

Heusser, C.J. 1998 (1997). Deglacial setting of the Southern Andes following the last glacial maximum: a short review. 25:89-103.

Iturraspe, R.J. \& J.A. Strelin 2002. Dinámica de procesos y geoformas vinculadas al retroceso del glaciar O'Higgins-Campo de Hielo Patagónico Sur, Chile. 30:13-24.

Koremblit, G. \& J.A. Forte Lay 1993 (1991). Contribución al estudio agroclimático del norte de Tierra del Fuego (Argentina). 20(1):25-134.

Mazzoni, E. 2001. Distribución espacial y caracterización geomorfológica de "Bajos sin Salida" de la Patagonia austral extracordillerana. 29:5-24.

Porter, C. \& A. Santana 2003. Rapid $20^{\text {th }}$ century retreat of ventisquero Marinelli in the Cordillera Darwin icefield. 31:17-26.

Prieto, X. 1990 (1988). Geología del Cuaternario del área de cabo Negro, estrecho de Magallanes, Chile. 18:35-41.

Prieto, X. 1990 (1988). Reconocimiento del Cuaternario entre Punta Arenas y río Chabunco, estrecho de Magallanes, Chile. 18:43-49.

Prieto, X. 1991 (1989-1990). El Terciario inferior de río Bueno y río Mayo, Tierra del Fuego, Magallanes, 19(1):51-56.

Prieto, X. 1994 (1992). Estratigrafía y ambientes de depositación durante el Cretácico inferior, seno Ultima Esperanza a seno Obstrucción, región de Magallanes. 21:71-83.

Prieto, X. \& M. Winslow 1994 (1992). El Cuaternario del estrecho de Magallanes. I. sector Punta Arenas-Primera Angostura. 21:85-95.
Santana, A. 1982. Resumen meteorológico año 1982. Estación "Jorge C. Schythe". 13:225-230.

Santana, A. 1983. Resumen meteorológico año 1983. Estación "Jorge C. Schythe". 14:133-138.

Santana, A. 1984. Variación de las precipitaciones de 97 años en Punta Arenas como índice de posibles cambios climáticos. 15:51-60.

Santana, A. 1984. Resumen meteorológico año 1984. Estación "Jorge C. Schythe". 15:61-66.

Santana, A. 1986 (1985-1986). Resumen meteorológico año 1985. Estación "Jorge C. Schythe". 16:91-97.

Santana, A. 1987. Resumen meteorológico año 1986. Estación "Jorge C. Schythe". 17:103-109.

Santana, A. 1990 (1988). Resumen meteorológico, año 1987. Estación "Jorge C. Schythe". (Latitud $53^{\circ} 08^{\prime}$ Sur; Longitud $70^{\circ} 53^{\prime}$ Oeste) (Altura: $6 \mathrm{~m}$ sobre el nivel del mar). 18:127134.

Santana, A. 1990 (1988). Resumen meteorológico, año 1988. Estación "Jorge C. Schythe". (Latitud $53^{\circ} 08^{\prime}$ Sur; Longitud 7053'Oeste) (Altura: $6 \mathrm{~m}$ sobre el nivel del mar). 18:135-142.

Santana, A. 1991 (1989-1990). El clima de Punta Arenas durante 1989. 19(1):81-91.

Santana, A. 1991 (1989-1990). El clima de Punta Arenas durante 1990. 19(1):93-97.

Santana, A. 1993 (1991). El clima de Punta Arenas durante 1991. 20(1):113-123.

Santana, A. 1994 (1992). El clima de Punta Arenas durante 1992. 21:147-157.

Santana, A. 1994 (1993-1994). Resumen meteorológico año 1993. Estación "Jorge C. Schythe". 22:79-86.

Santana, A. 1995. Resumen meteorológico año 1994. Estación "Jorge C. Schythe". 23:119-126.

Santana, A. 1996. Resumen meteorológico año 1995. Estación "Jorge C. Schythe". 24:97-104.

Santana, A. 1998 (1997). Resumen meteorológico año 1996. Estacción "Jorge C. Schythe". 25:127-134.

Santana, A. 1998. Resumen meteorológico año 1997. Estación "Jorge C. Schythe" (5308'S; 7053’W; 6 m s.n.m.). 26:141-149.

Santana, A. 1999. Resumen meteorológico año 1998. Estación "Jorge C. Schythe" (5308'S; 7053’W; 6 m s.n.m.). 27:117-124.

Santana, A. 2000. Resumen meteorológico año 1999. Estación "Jorge C. Schythe”. 28:143:150. 
Santana, A., J. Calderón \& N. Butorovic 2001. Características climatológicas del área de bahía Laredo, Magallanes, Chile. 29:5568.

Santana, A., C. Porter, N. Butorovic \& C. Olave 2006. Primeros antecedentes climatológicos de Estaciones Automáticas (AWS) en el canal Beagle, Magallanes, Chile. 34:5-20.

Santana, A. 2006. Resumen meteorológico año 2005. Estación "Jorge C. Schythe" (530ㅇ's; 7053’W; 6 m s.n.m.). 34:81-90.

Santana, A., C. Porter, N. Butorovic \& C. Olave. 2007. Características climáticas del canal Brecknock en los 5430'S de latitud, Magallanes, Chile. 35(1):5-18.

Santana, A. 2007. Resumen meteorológico año 2006. Estación "Jorge C. Schythe" (5308'S; 7053’O; 6 m s.n.m.). 35(1):81-90.

Skewes, M.A. 1978. Geología, petrología, quimismo y origen de los volcanes del área Pali-Aike, Magallanes, Chile. 9:95-106.

Suárez, M. 1976. La cordillera patagónica; su división y relación con la península Antártica. 7:105-113.

Tuhkanen, S., I. Kuokka, J. Hyvönen, S. Stenroos \& I. Niemelä 1992 (1989-1990). Tierra del Fuego as a target for biogeographical research in the past and present. 19(2):5-107.

Uribe, P. 1980. Estratigrafía y petrografía de la formación Hardy en bahía Scourfield, isla Wollaston, Magallanes, Chile. 11:139-150.

Uribe, P. 1981. Origen y geomorfología de la punta Dungeness, Patagonia. 12:143-158.

Uribe, P. 1982. Desglaciación en el sector central del estrecho de Magallanes: consideraciones geomorfológicas y cronológicas. 13:103111.

Wellman, R.W. 1972. Origen de la cueva del Milodón en Última Esperanza. 3(1-2):97-101.

Zamora, E. 1977. Resumen meteorológico 19721977. Estación "Jorge Ch. Schythe". Instituto de la Patagonia. (Latitud $53^{\circ} 08^{\prime}$ Sur; Longitud 7053'Oeste) (Altura: $4 \mathrm{~m}$ sobre el nivel del mar). 8:391-399.

Zamora, E. 1978. Resumen meteorológico año 1978. Estación "Jorge. C. Schythe". Instituto de la Patagonia. (Latitud $53^{\circ} 08^{\prime}$ Sur; Longitud 7053'Oeste) (Altura: $4 \mathrm{~m}$ sobre el nivel del mar). 9:233-236.
Zamora, E. 1979. Resumen meteorológico año 1979. Estación "Jorge C. Schythe". Instituto de la Patagonia. (Latitud $53^{\circ} 08^{\prime}$ Sur; Longitud 7053'Oeste) (Altura: $4 \mathrm{~m}$ sobre el nivel del mar). 10:245:250.

Zamora, E. \& A. Santana 1979. Características climáticas de la costa occidental de la Patagonia entre las latitudes $46^{\circ} 40^{\prime}$ y $56^{\circ} 30^{\prime}$ S. 10:109-144.

Zamora, E. \& A. Santana 1979. Oscilaciones y tendencias térmicas en Punta Arenas entre 1888 y 1979 . 10:145-154.

Zamora, E. \& A. Santana 1980. Régimen térmico y comportamiento pluviométrico en Punta Arenas: 10 años de registro en la estación "Jorge C. Schythe". 11:121-138.

Zamora, E. 1980. Resumen meteorológico año 1980. Estación "Jorge C. Schythe". (Latitud 530' Sur; Longitud 7053’Este) (Altura: 4 m sobre el nivel del mar). 11:335-342.

Zamora, E. \& A. Santana 1981. Resumen meteorológico. Año 1981. Estación "Jorge C. Schythe”. (Latitud: 5308'Sur; Longitud: 7053'Oeste (Altura: $6 \mathrm{~m}$ sobre el nivel del mar). 12:301-306.

\section{ZOOLOGÍA}

Aguayo, A., J. Acevedo, C. Valenzuela \& C. Venegas 2001. Censo de albatros de ceja negra Diomedea melanophris Temmink, 1828, en las islas Ildefonso y comentarios sobre su nidificación en isla Evout. 29:165-172.

Aguayo, A., J. Acevedo \& P. Acuña 2003. Nuevo sitio de anidamiento del albatros ceja negra, Diomedea melanophris Temmink 1828, en el seno Almirantazgo, Tierra del Fuego, Chile. 31:91-96.

Anderson, C. \& R. Rozzi 2000. Avian communities in the Southernmost forests of the world: methodological variations for determining species composition. 28:89-100.

Anderson, C.B. \& P.F. Hendrix 2002. Hallazgo de Eiseniella tetraedra (Savigny 1826) (Annelida: Oligochaeta) en isla Navarino, Chile. 30:143-146.

Angulo, A. 1976. Salpis antennata Mabille y Salpis virgata Rindge: nuevos para Magallanes, Chile (Lepidoptera: Geometridae). 7:195-196. 
Angulo, A. 1976. Trichophthalma jaffueli Stuardo: nuevo para Magallanes, y algunas consideraciones sistemáticas sobre Trichophthalma amaena Bigot y Eurygastromyia sexmaculata (Edwards) (Diptera: Nemestrinidae). 7:197-199.

Angulo, A. \& T.S. Olivares 1993 (1991). Microestructura del exocorion en huevos de algunas especies de nóctuidos (Lepidoptera: Glossata: Noctuidae). 20(1):95-100.

Astorga, M.S., S. Flores \& T. Soto 2002. Contenido de lípidos y proteínas en huevos y estados larvales de centolla ((Lithodes santolla Mol.) y centollón (Paralomis granulosa Jac.) (Crustacea: Decapoda). 30:117-121.

Atalah, A. 1975. Presencia de Chaetophractus villosus (Edentata: Dasypodidae). Nueva especie para la región de Magallanes, Chile. 6(1-2):169-171.

Atalah, A. \& W. Sielfeld 1976. Presencia de Batrachyla antartandica Barrio en Magallanes. 7:169-170.

Balbontín, F., I. Campodonico \& L. Guzmán 1979. Descripción de huevos y larvas de especies de Careproctus (Pisces: Liparidae) comensales de Paralomis granulosa y Lithodes antarctica (Crustacea: Lithodidae). 10:235-243.

Barros, A. 1971. Aves observadas en las islas Picton, Nueva, Lennox y Navarino Oriental. 2(1-2):166-180.

Barros, A. 1976. Nuevas aves observadas en las islas Picton, Nueva, Lennox y Navarino oriental. 7:189-193.

Brncic, D. 1980. Los géneros Scaptomyza Hardy y Drosophila Fallen (Diptera, Drosophilidae) en la región de Magallanes. 11:293-299.

Campodonico, I. 1971. Desarrolllo larval de la centolla Lithodes antarctica Jacquinot, en condiciones de laboratorio (Crustacea, Decapoda, Anomura: Lithodidae). 2(1-2):181-190.

Campodonico, I. \& L. Guzmán 1972. Lithodes murrayi Henderson, 1888, nuevo litódido para la Patagonia austral de Chile (Crustacea, Decapoda, Anomura). 3(1-2):221-232.

Campodonico, I. \& L. Guzmán 1972. Desarrollo larval de Eurypodius latreillei Guérin en condiciones de laboratorio (Crustacea Brachyura: Majidae, Inachinae). 3(1-2):233-247.
Campodonico, I. \& L. Guzmán 1973. Contribución a la biología de Acanthocyclus albatrossis Rathbun, 1898. 4(1-3):373-416.

Campodonico, I., L. Guzmán \& A. Sanhueza 1974. Madurez sexual en los machos de la centolla Lithodes antarctica Jacquinot, del área Punta Arenas-Porvenir, estrecho de Magallanes. 5(1-2):215-222.

Campodonico, I. 1978. Un caso de inversión en la asimetría de las hembras de Paralomis granulosa (Jacquinot) (Decapoda, Anomura, Lithodidae). 9:231-232.

Campodonico, I. 1979. La veda de la centolla ( $\mathrm{Li}$ thodes antarctica Jacquinot). 10:229-234.

Capella, J., J. Gibbons \& Y. Vilina 1999. La orca, Orcinus orca (Delphinidae) en aguas chilenas entre Arica y el cabo de Hornos. 27:63-72.

Cariceo, Y., E. Mutschke \& C. Ríos 2002. Ensambles de Isopoda (Crustacea) en discos de fijación del alga Macrocystis pyrifera (C. Agardh) (Phaeophyta) en el estrecho de Magallanes, Chile. 30:83-94.

Cekalovic, T. 1972. Descripción de la larva y pupa de Agriotes australis Fairm, 1883 (Coleoptera - Elateridae). 3(1-2):203-208.

Cekalovic, T. \& A.E. Quezada 1973. Descripción de la larva, pupa y distribución geográfica de Emmallodera multipunctata curvidens Kulzer, 1955 (Coleoptera - Tenebrionidae). 4(1-3):417-422.

Cekalovic, T. 1981. Descripción de larvas, observaciones sobre hábitat y distribución geográfica de Pycnochila fallaciosa (Chevrolat), 1854 (Coleoptera, Cicindelidae). 12:251-255.

Conti, E., G. Costa, A. Petralia \& A. Sobral 1994 (1993-1994). Eco-ethological research on the Antarctic and Subantarctic fauna. 4. Biology of Taurocerastes patagonicus Phil. (Coleoptera, Geotrupidae). 22:69-78.

Couve, E. \& C. Vidal 1999. Tórtola cordillerana, Metriopelia melanoptera (Molina 1782), una nueva especie de ave para la región de Magallanes. 27:115-116.

Di Russo, C., L. de Pasquale \& M. Rampini 1996. Preliminary observations on the biology of two Patagonian Rhaphidophoridae (Orthoptera): Heteromallus cavicola and Udenius w-nigrum. 24:105-116. 
Duret, J.P. 1977. Dos nuevas Mycetophila de Magallanes, Chile (Diptera, Nematocera). 8:349-353.

Duret, J.P. 1977. Lista previa de los Mycetophilidae de Monte Alto, Magallanes, Chile (Diptera, Nematocera). 8:355-361.

Duret, J.P. 1979. El género Mycetophila en la Patagonia. II. Ocho especies nuevas de Magallanes, Chile (Diptera, Mycetophilidae). 10:219-228.

Duret, J.P. 1980. El género Mycetophila en la Patagonia (Diptera, Mycetophilidae). IV. Descripción de diecinueve especies nuevas. 11:301-317.

Duret, J.P. 1981. El género Mycetophila en la Patagonia (Diptera, Mycetophilidae). VI. Descripción de quince especies nuevas. 12:239-250.

Duret, J.P. 1984. Los micetofílidos del archipiélago cabo de Hornos. 15:37-48.

Faúndez, E.I. 2007. Lista de las especies de Acanthosomatidae (Insecta: Hemiptera) de la región de Magallanes (Chile). 35(1):75-78.

Faúndez, E.I. 2007. Datos sobre las especies del género Steatoda Sundevall, 1833 (Arachnida; Theridiidae) de la región de Magallanes (Chile). 35(1):79-80.

Faúndez, E.I. 2007. Primeros registros para la región de Magallanes de las avispas sesiliventres Ametastegia glabrata (Fallen, 1808) y Periclista dapotoae Smith, 2002 (Hymenoptera: Symphyta: Tenthredinidae). 35(2):59-60.

Gazitúa, F., J. Gibbons \& J. Cárcamo 1999. Primer registro de delfín cruzado, Lagenorhynchus cruciger (Delphinidae), para el estrecho de Magallanes. 27:73-92.

Gibbons, J., J. Capella, R. Matus \& L. Guzmán 1998. Presencia de la ballena jorobada, Megaptera novaeangliae (Balaenopteridae), en los canales patagónicos de Chile. 26:69-75.

Gibbons, J. F. Gazitúa \& C. Venegas 2000. Cetáceos en el estrecho de Magallanes y senos Otway, Skyring y Almirantazgo. 28:107-119.

Gibbons, J. \& C. Miranda 2001. Presencia del elefante marino del Sur (Mirounga leonina) (Phocidae) en seno Almirantazgo, Tierra del Fuego. 29:157-159.

Gibbons, J. J.J. Capella \& Y.A. Vilina 2004. Ventral fluke pigmentation of humpback whale Megaptera novaeangliae population at the
Francisco Drake Marine Park, Straits of Magellan, Chile. 33:63-67.

Gibbons, J., J.J.Capella, A. Kusch \& J. Cárcamo 2006. The southern right whale Eubalaena australis (Desmoulins, 1822) in the strait of Magellan, Chile. 34:75-80.

Gibbons, J., Y.A. Vilina \& J. Cárcamo 2007. Distribución y abundancia de cisne coscoroba (Coscoroba coscoroba), cisne de cuello negro (Cygnus melancoryphus) y del flamenco chileno (Phoenicopterus chilensis) en la región de Magallanes. 35(2): 53-58.

Guzmán, L. \& I. Campodonico 1972. Fecundidad de la centolla Lithodes antarctica Jacquinot (Crustacea, Decapoda, Anomura). 3(1-2):249258.

Guzmán, L. \& I. Campodonico 1973. Presencia de Chelonia mydas agassizi Bocourt en Magallanes. 4(1-3):339-341.

Guzmán, L. \& I. Campodonico 1973. Algunos aspectos de la biología de Eleginops maclovinus (Cuv. \& Val.), 1830, con especial referencia a su morfometría, caracteres merísticos y alimentación. 4(1-3):343-371.

Guzmán, L. \& I. Campodonico 1976. Notorhynchus cepedianus (Peron, 1087), en la entrada oriental del estrecho de Magallanes (Elasmobranchii, Hexanchidae). 7:207-210.

Guzmán, L. 1978. Patrón de distribución espacial y densidad de Nacella magellanica (Gmelin, 1791) en el intermareal del sector oriental del estrecho de Magallanes (Mollusca, Gastropoda). 9:205:219.

Guzmán, L. 1978. Registro de Cetorhinus maximus (Gunner, 1965) en aguas de Chile austral (Elasmobranchii: Cetorhinidae). 9:229-230.

Guzmán, L., A. Atalah \& C. Venegas 1986 (19851986). Composición específica y estructura de la comunidad de aves de verano en el complejo de la tundra magallánica. 16:75-86.

Guzmán, L. \& C. Ríos 1987. Alometría en Lithodes antarcticus Jacquinot, 1853 (Crustacea: Decapoda): largo de la quela derecha versus largo del caparazón. 17:89-98.

Hromic, T. 1990 (1988). Presencia de Antarcticella (Candeina) antarctica (Leckie \& Webb) (Protozoa, Foraminiferida, Candeinidae) en la cuenca austral. 18:87-97. 
Hromic, T. 1991 (1989-1990). Planorotalites australiformis (Jenkins, 1965) (Foraminiferida: Globorotaliidae) en el Eoceno de la cuenca austral de Chile. 19(1):57-65.

Hromic, T. 1993 (1991). Foraminíferos de la formación Brush Lake: Cuenca Austral, Chile. 20(1):101-111.

Hromic, T. \& H. Águila 1994 (1993-1994). Asociación de foraminíferos epibiontes, bahía Zenteno, estrecho de Magallanes, Chile. 22:51-61.

Hromic, T. 1995. Catálogo de Foraminíferos (Protozoa: Foraminiferida) del Terciario de Chile. 23:51-94.

Hromic, T. 1996. Foraminíferos bentónicos (Protozoa: Foraminiferida) de aguas profundas del estrecho de Magallanes. 24:65-86.

Hromic, T. 1998. Foraminiferida: Miliolina del estrecho de Magallanes, Chile: frecuencia y distribución en el área subantártica y antártica. 26:101-106.

Hromic, T. 1999. Foraminíferos bentónicos de canales australes: canal Kirke, golfo Almirante Montt y seno Última Esperanza, XII ${ }^{a}$ Región, Magallanes y Antártica Chilena. 27:91-104.

Hromic, T. 2001. Foraminíferos bentónicos del canal Baker $\left(47^{\circ} \mathrm{S}-74^{\circ} \mathrm{W}\right)$, Pacífico sudoriental, Chile. 29:135-156.

Hromic, T. 2002. Foraminíferos bentónicos de bahía Nassau, cabo de Hornos, Chile. Comparación con foraminíferos del cono Sur de América, Antártica e islas Malvinas. 30:95-108.

Hromic, T. \& M. Zúñiga 2003. Foraminíferos (Protozoa: Foraminiferida) de la superfamilia Buliminacea Jones 1875, en canales y fiordos patagónicos, Chile. 31:55-74.

Hromic, T., C. Andrade, I. Ramírez \& S. Vidal 2004. Nuevo registro de Spirillina tuberculata Brady (Protozoa: Foraminiferida) en aguas chilenas. 32:35-41.

Imberti, S. 2005. Distribución otoñal de aves marinas y terrestres en canales chilenos. 33:21-30.

Jory, J.E., C. Venegas \& W.A. Texera 1974. La avifauna del Parque Nacional "Laguna de los Cisnes", Tierra del Fuego, Chile. 5(12):131-154.

Jory, J.E. 1975. Observaciones etológicas en Pterocnemia pennata pennata (d'Orbigny) (Aves: Rheidae). 6(1-2):147-159.
Jory, J.E. \& W.A. Texera 1975. Anas bahamensis rubrirostris (pato gargantillo) en Magallanes, Chile. 6(1-2):161-162.

Kusch, A., \& M. Marín 2002. Distribución del chorlo de campo, Oreopholus ruficollis (Wagler) (Charadriidae) en Chile. 30:133-142.

Kusch,A. \& M. Marín 2004. Distribución del chorlo chileno, Charadrius modestus (Lischtenstein) (Charadriidae) en Chile. 32:69-78.

Kusch, A., M. Marín, D. Oheler \& S. Drieschman 2007. Notas sobre la avifauna de isla Noir

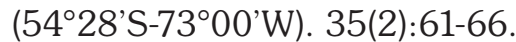

Lanfranco, D. 1974. Contribución al conocimiento de la Ichneumofauna de la región de Magallanes (Hymenoptera; Ichneumonidae). 5(1-2):199-208.

Lanfranco, D. 1977. Entomofauna asociada a los bosques de Nothofagus pumilio (Poepp. et Endl.) Krasser en la región de Magallanes. I Parte: Monte Alto (río Rubens, Última Esperanza). 8:319-348.

Lanfranco, D. 1980. Estudios entomofaunísticos en el archipiélago del cabo de Hornos. 11:281-291.

Lanfranco, D. 1981. Estudios entomofaunísticos en el archipiélago del cabo de Hornos. 2. Prospección preliminar de suelo-superficie en surgidero Romanche (isla Bayly). 12:229-238.

Lanfranco, D. 1982. Presencia de Andiperla willinki en Chile. 13:207-209.

Lanfranco, D. 1983. Estudios entomofaunísticos en el archipiélago del Cabo de Hornos. III. Composición y estructura de suelo-superficie asociada a bosques y turbales en caleta Toledo (isla Deceit: 5549'S-6706'O). 14:119-131.

Lanfranco, D. 1993 (1991). Sinopsis de los insectos que atacan bosques de lenga [Nothofagus pumilio (Poepp. \& Endl.)] Krass. en Magallanes. 20(1):89-83.

Langley, S., L. Guzmán \& C. Ríos 1980. Aspectos dinámicos de Mytilus chilensis (Hupé, 1840) en el estrecho de Magallanes. I. Distribución, densidad y disposición espacial en el intermareal. 11:319-332.

Latorre, C. 1998. Paleontología de Mamíferos del alero Tres Arroyos I. Tierra del Fuego, XII Región, Chile. 26:77-90.

López, B. \& E. Domínguez 2002. Presencia de Strix rufipes King 1818 en el Parque Histórico 
Rey Don Felipe, península de Brunswick, Magallanes. 30:147-150.

Marchant, M. 1991 (1989-1990). Bibliografía micropaleontológica de Chile: Foraminíferos del Terciario. 19(1):67-80.

Marín, M. \& E. Couve 2001. La gaviota de Franklin, Larus pipixcan Wagler, 1831 (Laridae), al sur de latitud $41^{\circ} \mathrm{S}$, con nuevos registros de distribución. 29:161-163.

Marín, M., A. Kusch, D. Oehler \& S. Drieschman 2006. Distribution, breeding and status of the striated caracara Phalcoboenus australis (Gmelin, 1788) in Southern Chile. 34:65-74.

Marín, M. \& D. Oehler 2007. Una nueva colonia de anidamiento para el albatros de ceja negra (Thalassarche melanophris) para Chile. 35(1):29-34.

Markham, B.J. 1970. Reconocimiento faunístico del área de los fiordos Toro y Cóndor, isla Riesco, Magallanes. 1(1):41-59.

Markham, B.J. 1970. Nota sobre las subespecies del culpeo (Dusicyon culpaeus) en Magallanes. 1(1):61-65.

Markham, B.J. 1970. Cuatro nuevas especies de aves para Magallanes, 1(1):67-70.

Markham, B.J. 1971. Censo invernal de cisnes y flamencos en Magallanes. 2(1-2):147-157.

Markham, B.J. 1971. Descripción de una nueva subespecie de "tordo", Curaeus curaeus recurvirostris , subsp. nov. 2(1-2):158-163.

Markham, B.J. 1971. Observaciones sobre el elefante marino del sur y el leopardo marino en la península Brecknock (Parque Nacional "Alberto M. de Agostini"), Tierra del Fuego. 2(1-2):160-163.

Markham, B.J. 1971. Presencia del "culpeo" (Dusicyon culpaeus) en la isla Hoste, Tierra del Fuego. 2(1-2):164-165.

Marshall, L.G. \& P. Salinas 1991 (1989-1990). Vertebrados continentales del Mioceno inferior de Magallanes, Chile. 19(1):27-38.

Martinic, M. 1982. Antecedentes para la historia del conocimiento botánico y zoológico de la región del estrecho de Magallanes. 13:113-123.

Matus, R. 1998. Presencia accidental de Corvus splendens (Aves: Corvidae) y nuevos registros de aves raras en Magallanes: Rollandia gallardoi y Eremobius phoenicurus. 26:137-139.
Matus, R. \& C. Barría 1999. Adiciones a la lista de aves del Parque Nacional Torres del Paine. 27:105-113.

McGehee, S.M., R. Rozzi, C. Anderson, S. Ippi, R. Vásquez \& S. Woodland 2004. Late-summer presence of the Patagonian tyrant Colorhamphus parvirostris (Darwin) on Navarino Island, Cape Horn County, Chile. 32:25-33.

Montiel, A., D. Gerdes \& C. Ríos. 2001. Distribución y abundancia del macrozoobentos en una microcuenca marina submareal del estrecho de Magallanes, Chile. 29:117-133.

Montiel, A., C. Ríos \& E. Mutschke 2007. Biodiversidad de los poliquetos sublitorales de las costas noroccidental y sur de la isla de Tierra del Fuego (Chile). 35(2):41-52.

Morrison, E. \& G. Pequeño 2003. Primer registro del zoárcido Crossostomus sobrali (Lloris \& Rucabado 1987) (Osteichthyes, Zoarcidae) en el estrecho de Magallanes, distrito Fueguino, Chile. 31:105-109.

Mutschke, C. Ríos \& A. Montiel 1998. Situación actual de la macrofauna presente en el intermareal de bloques y cantos de bahía Laredo, estrecho de Magallanes. 26:5-29.

Mutschke, E., C. Ríos, M. Santana \& E. Estay 2000. Características y variabilidad abiótica y biótica en un sistema lótico de seno Skyring, región de Magallanes. 28:127-138.

Pastene, L.A. \& H. Shimada 1999. Report of a sighting survey in Chile's exclusive economic zone with comments on Sei whale distribution. 27:51-62.

Pérez, V. 1994 (1992). Bibliografía Entomológica Chilena: Hymenoptera III. 21:103-110.

Pérez, V. \& E. Mutschke 1994 (1993-1994). Las especies de Aeshna Fabricius (Odonata: Anisoptera: Aeshnidae) en la región de Magallanes. 22:63-68.

Pérez, V. 1995. Primer registro de la familia Chrysididae (Hymenoptera) en la región de Magallanes. 23:95-100.

Pérez, V. 1996. Lista de las especies de mariposas diurnas (Lepidoptera: Rhopalocera) de Magallanes y clave para su identificación. 24:49-64.

Pérez, V., E. Mutschke \& M. Vera 1998 (1997). Hexápodos (Arthropoda: Parainsecta e Insecta) en un proceso de desglaciación y revegetación en Fuego-Patagonia (Chile). 25:57-76. 
Pérez, V. 1998. Moscas abeja (Diptera: Bombylidae) de Magallanes: lista preliminar, distribución geográfica, relaciones zoogeográficas y ecología. 26:101-106.

Pérez, V. 1999. Hypodynerus guaicuru n.sp. (Hymenoptera: Vespidae), una avispa albañil de la región de Magallanes. 27:83-89.

Pérez, V. 2000. Las especies de véspidos (Hymenoptera: Vespidae) de la región de Magallanes: clave de identificación, distribución y relaciones zoogeográficas. 28:119-126.

Pérez, V. 2000. La avispa "chaqueta amarilla", Vespula germanica (Fabricius) (Hymenoptera: Vespidae), en la región de Magallanes. 28:139-142.

Pérez, V. 2002. Sobre Cercophana frauenfeldi Felder (Lepidoptera: Cercophanidae) en la región de Magallanes. 30:113-116.

Pérez, V. 2002. Sobre los términos taxonomía y taxón en idioma castellano. 30:151-156.

Pérez, V. 2003. Primer registro de Conognatha germaini Théry (Coleoptera: Buprestidae) en la región de Magallanes. 31:111-113.

Pérez, V. 2004. El Dr. Rodulfo Amando Philippi (1808-1904): su contribución al conocimiento de la biodiversidad en insectos de Chile. 32:43-53.

Pérez, V., E. Faúndez, D. Vargas, A. Zúñiga \& N. Butorovic 2005. El regreso de la mariposa colorada Cynthia carye (Hübner, 1812) (Lepidoptera: Nymphalidae) a Punta Arenas, región de Magallanes. 33:37-40.

Pérez, V. 2005. Agrius cingulatus (Hübner, 1839) e Hyles lineata (Fabricius, 1775) (Lepidoptera: Spingidae), primeros registros de esfíngidos para la región de Magallanes. 33:59-61.

Pérez, V. 2007. Avispas arañeras (Hymenoptera: Pompilidae) de la región de Magallanes: nuevos registros, clave de identificación de especies y distribución geográfica. 35(2):21-28.

Petersen, J. 1990 (1988). Chilecomadia valdiviana (Philippi) (Lepidoptera: Cossidae), asociado a Nothofagus pumilio (Poepp, et Endl.) Krasser (lenga) en la región de Magallanes. 18:51-55.

Pine, R.H. 1973. Una nueva especie de Akodon (Mammalia: Rodentia: Muridae) de la isla Wellington, Magallanes, Chile. 4(1-3):423426.
Pisano, E. 1977. Contribución de Enrique Ibar Sierra al conocimiento de la naturaleza de la Patagonia oriental austral. 8:95-119.

Raedeke, K.J. 1976. La inmovilización de guanacos (Lama guanicoe) con cloruro de Succinilcolina. 7:185-188.

Rau, J., J. Yáñez \& F. Jaksic 1978. Confirmación de Notyomis macronyx alleni O. y Eligmodontia typus typus C., y primer registro de Akodon (Abrothrix) lanosus T. (Rodentia: Cricetidae) en la zona de Última Esperanza (XII Región, Magallanes). 9:203-204.

Retamal, M.A. 1994 (1992). Los Lithodidae chilenos. 21:111-129.

Ríos, C. C. Noziglia \& L. Guzmán. 1987. Desarrollo larvario de los gastrópodos Siphonaria (Pachysiphonaria) lessoni (Blainville, 1824) y Kerguelenella lateralis (Gould, 1846) (Pulmonata: Siphonariidae) del estrecho de Magallanes, Chile. 17:77-88.

Ríos, C. \& D. Gerdes 1998 (1997). Ensamble bentónico epifaunístico de un campo intermareal de bloques y cantos en bahía Laredo, estrecho de Magallanes. 25:47-55.

Ríos, C., E. Mutschke \& Y. Cariceo 2003. Estructura poblacional de Pseudechinus magellanicus Philippi 1857) (Echinoidea: Temnopleuridae) en grampones de la macroalga sublitoral Macrocystis pyrifera (L.) C. Agardh en el estrecho de Magallanes. 31:75-86.

Rozzi, R. \& M. Sherrifs 2003. El visón (Mustela vison Schreber, Carnivora: Mustelidae), un nuevo mamífero exótico para la isla Navarino. 31:97-104.

Santana, M. 1998. Estudios en el comportamiento reproductivo en la naturaleza y desarrollo intracapsular en laboratorio del caracol Trophon geversianus (Gastropoda: Muricidae). 26:31-40.

Schiavini, A., E. Frere, P. Yorio \& A. Parera 1999. Las aves marinas de la isla de Los Estados, Tierra del Fuego, Argentina: revisión histórica, estado poblacional y problemas de conservación. 27:25-40.

Schlatter, R.P., P. Vergara \& M. Briones 2002. El canquén (Chloephaga polyocephala: Anatidae) en bosques de Tierra del Fuego: distribución y depredadores. 30:61-66.

Shapiro, A.M. 1998 (1997). Impactos antropogénicos sobre la fauna de mariposas (Lepidoptera: 
Rhopalocera) de Patagonia austral y Tierra del Fuego. 25:117-126.

Sielfeld, W. 1976. Presencia de Exomegas macrostenus (Burmeister) (Myxini: Petromizonidae) en aguas magallánicas. 7:211-213.

Sielfeld, W. 1976. Presencia del género Archiropsetta Norman, 1930 (Pisces: Pleuronectiformes) en aguas patagónicas chilenas. 7:215-217.

Sielfeld, W. 1977. Reconocimiento macrofaunístico terrestre en el área de seno Ponsonby (isla Hoste). 8:275-298.

Sielfeld, W., C. Venegas \& A. Atalah. Apéndice: M. Martinic 1977. Consideraciones acerca del estado de los mamíferos marinos en Chile. 8:295-315.

Sielfeld, W. \& C. Venegas 1978. Observaciones de delfínidos en los canales australes de Chile. 9:153-156.

Sielfeld, W. 1978. Algunas consideraciones sobre fócidos (Pinnipedia) asociados a las costas de Chile. 9:153-156.

Sielfed, W., C. Venegas, A. Atalah \& J. Torres 1978. Prospección de otáridos en las costas de Magallanes. 9:157-169.

Sielfeld, W. 1979. Consideraciones acerca de tres especies de Mesoplodon Gervais (Cetacea: Ziphiidae) presentes en las aguas chilenas. 10:179-187.

Sielfeld, W. 1979. Nuevo registro de Netuma barbus (Lacepede) 1803 para aguas chilenas (Pisces: Siluriformes). 10:187-192.

Sielfeld, W. 1979. Consideraciones acerca de los pejesapos (Gobiesociformes: Pisces) conocidos para las costas magallánicas. 10:193-195.

Sielfeld, W. 1979. Presencia de Microcavia australis (G. y D'O.) en Magallanes (Mammalia: Caviidae). 10:197-199.

Sielfeld, W. 1980. Mamíferos marinos en colecciones y museos de Chile. 11:273-280.

Sielfeld, W., G. Guzmán \& N. Amado 2006. Distribución de peces del litoral rocoso de los canales patagónicos occidentales ( $48^{\circ} 37^{\prime} \mathrm{S}$ 533'ㅇ). 34:21-32.

Tapia, P. 1995. Patrón de actividad temporal de Abrothrix xanthorhinus (Waterhouse) en el sector de "Laguna Amarga", Parque Nacional Torres del Paine. 23:47-50.

Texera, W.A. 1972. Distribución y diversidad de mamíferos y aves en la provincia de Magallanes,
I. Análisis preliminar de la diversidad ecológica y variación taxonómica. 3(1-2):171-200.

Texera, W.A. 1973. Distribución y diversidad de mamíferos y aves en la provincia de Magallanes, II. Algunas notas ecológicas sobre los canales patagónicos. 4(1-3):291-305.

Texera, W.A. 1973. Distribución y diversidad de mamíferos y aves en la provincia de Magallanes, III. Historia geológica de los géneros de mamíferos nativos terrestres. 4(1-3):307-319.

Texera, W.A. 1973. Distribución y diversidad de mamíferos y aves en la provincia de Magallanes, IV. Zoogeografía de mamíferos nativos terrestres. 4(1-3):321-333.

Texera, W.A. 1973. Zaedyus pichiy (Edentata, Dasypodidae), nueva especie en la provincia de Magallanes, Chile. 4(1-3):335-337.

Texera, W.A. 1974. Algunos aspectos de la biología del huemul (Hippocamelus bisulcus) (Mammalia: Artyodactyla: Cervidae) en cautividad. 5(1-2):155-188.

Texera, W.A. 1974. Nuevos antecedentes sobre mamíferos de Magallanes. 5(1-2):189-198.

Texera, W.A. 1975. Descripción de una nueva subespecie de Ctenomys magellanicus (Mammalia; Rodentia; Ctenomyiidae) de Tierra del Fuego, Magallanes, Chile. 6(12):163-167.

Thatje, S. \& R. Bacardit 2001. Two zoeal stages of Betaeus truncatus (Dana 1852) (Decapoda: Caridea: Alpheidae) from Argentine coastal waters. 29:95-104.

Thatje, S. 2003. Review of the Thalassinidae (Crustacea: Decapoda) from Chile and Argentina. 31:115-122.

Torres, E.R., A. Manero \& F.A. Vargas 2006. Listado sistemático y distribución temporal de los peces del estuario del río Gallegos (Santa Cruz, Argentina). 34:59-64.

Traba, R. \& C. Ríos 1986 (1985-1986). Nota sobre la dieta de Salmo trutta (L.) en Tierra del Fuego, Magallanes. 16:87-90.

Venegas, C. \& P. Drouilly 1972. Nota aclaratoria acerca de la presencia de Parabuteo unicinctus unicinctus (Temminck) en Magallanes. 3(1-2)201-202.

Venegas, C. 1973. La garza mora (Ardea cocoi Linné) en Magallanes. 4(1-3):275-279. 
Venegas, C. 1973 El ostrero americano Haematopus ostralegus ssp. en el estrecho de Magallanes. 4(1-3):281-289.

Venegas, C. \& J.E. Jory 1974. Tres nuevas especies de aves para la región de Magallanes. 5(1-2):127-130.

Venegas, C. 1975. Dos adiciones de la fauna avial magallánica Bubulcus ibis (Ardeidae) y Agelaius thilius (Icteridae). 6(1-2):141-145.

Venegas, C. 1976. Observaciones ornitológicas en la tundra magallánica. I. Recuento descriptivo del área y de las observaciones aviales entre los paralelos $51^{\circ} 31^{\prime}$ 'S y $52^{\circ} 09^{\prime} \mathrm{S}$. 7:171-184.

Venegas, C. 1977. Extensión del rango meridional de la rara (Phytotoma rara Mol.) hasta Magallanes (Aves: Phytotomidae). 8:317-318.

Venegas, C. \& W. Sielfeld 1978. Registros de Mesoplodon layardii y otros cetáceos en Magallanes. 9:171-177.

Venegas, C. 1978. Pingüinos de barbijo (Pygoscelis antarctica) y macaroni (Eudyptes chrysolophus) en Magallanes. 9:179-183.

Venegas, C. \& W. Sielfeld 1979. Antecedentes para la determinación de un nuevo distrito zoogeográfico en el litoral exterior de Magallanes. 10:201-208.

Venegas, C. \& W. Sielfeld 1980. Un varamiento masivo de ballenas piloto (Globicephala melaena Traill) en Magallanes. 11:239-246.

Venegas, C. 1981. Aves de las islas Wollaston y Bayly, archipiélago del cabo de Hornos. 12:213-219.

Venegas, C. 1982. Nuevos registros ornitológicos en Magallanes. 13:183-187.

Venegas, C. 1982. Suplemento a la Guía de Campo para las Aves de Magallanes. 13:189-206.

Venegas, C. 1986 (1985-1986). Una prospección aérea de gansos sudamericanos (Chloephaga) en la estepa central de Magallanes. 16:75-86.

Venegas, C. \& A. Atalah 1987. Prospeccion aérea otoñal de tuninas overas (Cephalorhynchus commersonii) en el estrecho de Magallanes. 17:69-75.

Venegas, C. 1993 (1991). Ensambles avifaunísticos estivales del archipiélago cabo de Hornos. 20(1):69-82.

Venegas, C. 1994 (1993-1994). Comunidades estivales de aves terrestres en isla Noir. 22:43-49.
Venegas, C. 1995. Variación diaria y estacional de la avifauna asociada al Aeropuerto de Punta Arenas. 23:35-45.

Venegas, C. 1996. Estimación de la densidad poblacional, mediante transectos aéreos en línea, de la tunina overa Cephalorhynchus commersonii en el estrecho de Magallanes. 24:41-48.

Venegas, C. 1998. Pingüinos crestados (Eudyptes chrysocome Forster 1781, E. chrysolophus Brandt 1837) y de Magallanes (Spheniscus magellanicus Forster 1781) en isla Noir, Chile. 26:59-67.

Venegas, C. 2000. Avifauna de un bosque de lenga (Nothofagus pumilio) intervenido y de uno no intervenido en Magallanes continental, Chile. 28:101-106.

Venegas, C., J. Gibbons, A. Aguayo, W. Sielfeld, J. Acevedo, N. Amado, J. Capella, G. Guzmán \& C. Valenzuela 2002. Distribución y abundancia de lobos marinos (Pinnipedia: Otariidae) en la región de Magallanes, Chile. 30:67-82.

Vera-Palacios, M.A., R. Briones \& V. Jerez 2005. Registros de efemerópteros (Insecta: Ephemeroptera) en el río San Pedro, región de Magallanes. 33:31-35.

Vera-Palacios, M. 2007. Ephemeroptera (Insecta) en la región de Magallanes. 35(1):35- 44.

Vuilleumier, F. 1993 (1991). Especiación en aves de Fuego-Patagonia chilena: estudios preliminares. 20(1):83-88.

Vuilleumier, F. 1994 (1992). Biogeografía de las aves altoandinas: ensayo sobre algunos aspectos de su evolución. 21:39-66.

Vuilleumier, F., A.P. Capparella \& I. Lazo. 1994 (1992). Extensión del rango de Asthenes pyrrholeuca (Aves: Furnariidae) en FuegoPatagonia. 21:67-69.

Vuilleumier, F. 1998. Avian diversity in Fuego-Patagonia. 26:41-57.

Yáñez, J., W. Sielfeld, J. Valencia \& F. Jaksic 1978. Relaciones entre la sistemática y la morfometría del subgénero Abrothrix (Rodentia: Cricetidae) en Chile. 9:185-197.

Zúñiga-Rival, M. 2006. Estudio preliminar de los foraminíferos bentónicos (Protozoa: Foraminiferida) de bahía Yendegaia, Tierra del Fuego, Chile. 34:33-40. 